\title{
RAB38 confers a poor prognosis, associated with malignant progression and subtype preference in glioma
}

\author{
HONGJUN WANG and CHUANLU JIANG \\ Department of Neurosurgery, The Second Affiliated Hospital of Harbin Medical University, \\ Harbin, Heilongjiang 150086, P.R. China
}

Received August 7, 2013; Accepted August 30, 2013

DOI: 10.3892/or.2013.2730

\begin{abstract}
RAB38 is a new member of the RAB small G protein family that regulates intracellular vesicle trafficking. RAB38 is expressed in melanocytes and it has been shown that a point mutation in the postulated GTP-binding domain of RAB38 is the gene responsible for human Hermansky-Pudlak syndrome. However, the prognostic and molecular features of tumors with RAB38 expression is still unclear, as well as glioma. Whole genome mRNA expression microarray data on 220 glioma samples from the Chinese Glioma Genome Atlas (CGGA) database (http://www.cgga.org.cn) was applied as discovery set. Each grade of glioma patients was analyzed by the Kaplan-Meier method. To determine the protein expression levels of RAB38, further 82 glioma tissues were stained by immunohistochemistry. Three additional datasets (TCGA, GSE16011 and Rembrandt) were obtained as validation sets. The functional annotation of RAB38 was analyzed by Gene Ontology (GO) analysis and Gene set variation analysis (GSVA) in 89 glioblastomas (GBMs). High RAB38 expression was mainly increased in high-grade gliomas, and high RAB38 expression also conferred high mortality of glioma in the CGGA cohort. RAB38 showed a mesenchymal subtype, G3 subtype and isocitrate dehydrogenase 1 (IDH1) wild-type preference. GO and GSVA analysis showed that RAB38 was significantly correlated with migration. These results were validated in other 3 datasets. The expression levels of RAB38 were significantly associated with grade progression as well as prognosis in gliomas. RAB38 is an important prognostic biomarker and potential therapeutic target in gliomas.
\end{abstract}

\section{Introduction}

Gliomas are the most common and aggressive type of primary brain tumors. Despite recent advances in treatment

Correspondence to: Dr Chuanlu Jiang, Department of Neurosurgery, The Second Affiliated Hospital of Harbin Medical University, 246 Xuefu Road, Harbin, Heilongjiang 150086, P.R. China

E-mail: jc16688jcl@163.com

Key words: RAB38, survival, migration, glioma by a combination of surgery and chemotherapy and/or radiotherapy, the prognosis of malignant glioma is still extremely poor. Gliomas can be divided into low grade glioma (LGG, including astrocytomas, oligodendrogliomas and oligoastrocytomas) and high grade glioma (HGG, including anaplastic astrocytomas, anaplastic oligodendrogliomas, anaplastic oligoastrocytomas and glioblastomas) depending on the malignancy. Glioblastoma (GBM) is WHO grade IV glioma, the median survival time of which is approximately only one year $(2,3)$. The increased invasive capacity of GBM cells may be part of the reason for the poor prognosis. Thus, research on invasion of glioma is important.

$\mathrm{RAB}$, ras-related gtp-binding protein, is a member of the small GTPase super family, it is located in specific subcellular organelles and plays an important role in cell secretion, endocytosis, signal transduction and development (4). In recent years, the role in the control of different aspects of tumor progression has emerged. In tumors, main roles of RAB GTPases were cell migration, invasion, proliferation, communication with stromal cells and the development of drug resistance. Several RABs such as RAB1b, RAB24, $\mathrm{RAB} 25, \mathrm{RAB} 27 \mathrm{a}$ and RAB27b are disregulated in many cancers (5-8). The function of RAB27a in glioma cell lines was also reported $(9,10)$. RAB38 is the gene responsible for oculocutaneous albinism (1), but the effect of RAB38 in tumors is still unclear.

In the present study, we investigated mRNA expression microarray data on 220 glioma samples from the Chinese Glioma Genome Atlas (CGGA) database as discovery set and 3 additional published datasets as validation sets. Through evaluating the RAB38 expression status, we obtained the prognostic value. The protein expression level was validated in another 82 glioma samples from the Chinese Glioma Tissue Database (CGTD) by immunohistochemistry. In all 4 datasets, we performed function annotation of RAB38 by GO analysis and GSVA, as a result, its correlation with cell migration mainly was found.

\section{Materials and methods}

Datasets. Whole genome mRNA expression microarray data and clinical information on 220 glioma samples of all grades from the Chinese Glioma Genome Atlas (CGGA) database (2) (http://www.cgga.org.cn) were obtained as 
discovery set and 369 glioma expression files from the Cancer Genome Atlas (TCGA) database (11) (http://cancergenome. nih.gov), the Repository for Molecular Brain Neoplasia Data (REMBRANDT, http://caintegratorinfo. nci.nih. gov/rembrandt) and GSE16011 data (12) (http://www.ncbi.nlm. nih.gov/geo/query/acc.cgi?acc=GSE16011) were obtained as validation sets.

Immunohistochemistry (IHC). Paraffin-embedded specimens were cut into $4 \mu \mathrm{m}$ sections and heated at $65^{\circ} \mathrm{C}$ for $30 \mathrm{~min}$. The sections were deparaffinized with xylenes and rehydrated. Sections were submerged into EDTA ( $\mathrm{pH}$ 8.0) and autoclaved for antigen retrieval, then treated with $3 \%$ hydrogen peroxide, followed by incubation with $1 \%$ FBS. Anti-RAB38 antibody (Antibodies; ABIN1390129) was added and incubated overnight at $4^{\circ} \mathrm{C}$. Horseradish peroxidase (HRP) labeled secondary antibody in the MaxVision ${ }^{\mathrm{TM}}$ HRP-Polymer antirabbit IHC kit (KIT-5930 Maixin Biology, Fuzhou, China) was applied and incubated for $30 \mathrm{~min}$ at room temperature, followed by 5 -min incubation at room temperature with DAB provided in the kit for color development. The sections were finally counterstained with haematoxylin and mounted with Permount (BIOS, Beijing, China). Results were visualized and photographed under a light microscope (Olympus BX-51; Olympus Optical). The proportion of positively stained tumor cells was graded as follows: 0 , no positive tumor cells; $1,<5 \%$ positive tumor cells; $2,5-20 \%$ positive tumor cells; and $3,>20 \%$ positive tumor cells. The intensity of staining was recorded on a scale of 0 (no staining), 1 (weak staining, light yellow), 2 (moderate staining, yellowish brown) and 3 (strong staining, brown). The staining index was calculated as follows: staining index $=$ staining intensity $\mathrm{x}$ tumor cell staining grade. High RAB38 expression was defined as a staining index score $\geq 4$, while low expression was defined as a staining index $<4$.

Gene Ontology (GO) analysis. After Pearson correlation analysis, Gene Ontology analysis of the positively correlated genes $(r>0.4 ; \mathrm{P}<0.01)$ were analyzed by DAVID (http://david. abcc.ncifcrf.gov/ho me.jsp).

Gene set variation analysis (GSVA). Gene set variation analysis with RAB38 expression was analyzed by GSVA package (13) of R (14). Gene list was obtained from the web (http:// amigo.geneontology.org/) (GO:0030335, positive regulation of cell migration and GO:0030336, negative regulation of cell migration).

Statistical analysis. For molecular subtype annotation of the 4 datasets, we applied prediction analysis of microarrays (PAM) as previously reported (2). Quantitative results are shown as mean \pm standard deviation. The difference of RAB38 expression was tested by the Student's t-test in microarray data and by the Chi-square test in IHC results. Overall survival time (OS) was calculated from the date of diagnosis until death or the last follow-up. The survival curve of patients with high or low expressed RAB38 was calculated with the Kaplan-Meier method and the difference was analyzed using the two-sided log-rank test. A P-value $<0.05$ was considered to indicate a statistically significant result. All the data analysis was
Table I. Clinical characteristics of the 220 glioma patients.

\begin{tabular}{lccc}
\hline & Grade II & Grade III & Grade IV \\
\hline Case number & 97 & 34 & 89 \\
Median age (years) & 38 & 39 & 46 \\
Male \% & 55.7 & 52.9 & 58.4 \\
Median KPS & 90 & 80 & 80 \\
Median OS (days) & ND & 633 & 420 \\
\hline
\end{tabular}

$\mathrm{ND}$, not determined.

performed in MATLAB, GraphPad Prism and R. Written informed consent was obtained from the patient for publication of the present report and any accompanying images. The study was performed with the approval of the Ethics Committee of the Capital Medical University and it was in compliance with the Helsinki Declaration.

\section{Results}

Characteristics of glioma patients. In 220 glioma patients, there were 97 WHO grade II gliomas (astrocytomas, oligodendrogliomas and oligoastrocytomas), 34 WHO grade III gliomas (anaplastic astrocytomas, anaplastic oligodendrogliomas and anaplastic oligoastrocytomas) and 89 WHO grade IV gliomas (GBMs). Clinical information [age, gender, preoperational Karnofsky performance scale (KPS) score and treatment] were obtained from medical records of the CGGA database (Table I).

The expression of RAB38 in high grade gliomas was higher than those of low grade. After analyzing of differently expressed genes in the discovery dataset, we found that RAB38 was in the list of genes significantly differently expressed between LGGs and HGGs. RAB38 expression increased along with grade progression of glioma both in CGGA and the other 2 validation datasets (Fig. 1).

The protein expression level of RAB38 was detected by IHC. Eighty-two glioma samples were used to detect the expression status of RAB38 by IHC (Fig. 2). These samples included 38 LGGs and 44 HGGs. RAB38 showed a higher expression level in HGGs than those in LGGs $(\mathrm{P}<0.01)$. Thus, RAB38 expression showed high grade glioma preference both in mRNA and in protein level.

RAB38 may be a prognostic marker in anaplastic gliomas and glioblastomas. In the 220 glioma patients of CGGA, 216 patients with integrated prognostic information were further analyzed by Kaplan-Meier. Although several P-values in survival analysis was no statistical significance, the expression of RAB38 can be divided into high and low expression with different percent survival of each grade in CGGA and the other two datasets (Fig. 3). There was a marginal significance in anaplastic gliomas in CGGA (Fig. 3B; $\mathrm{P}=0.056$ ), but there was a significant different prognosis in anaplastic gliomas 

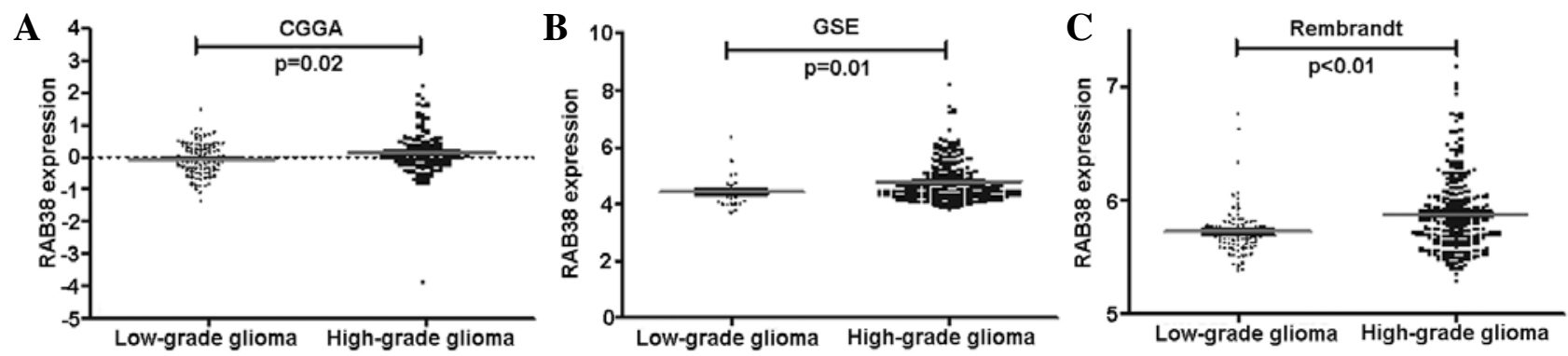

Figure 1. The expression difference of RAB38 in LGGs and HGGs of CGGA and the other two validation datasets. The expression of RAB38 was significant higher in HGGs than those of LGGs. (A) CGGA dataset, (B) GSE16011 dataset and (C) Rembrandt dataset.

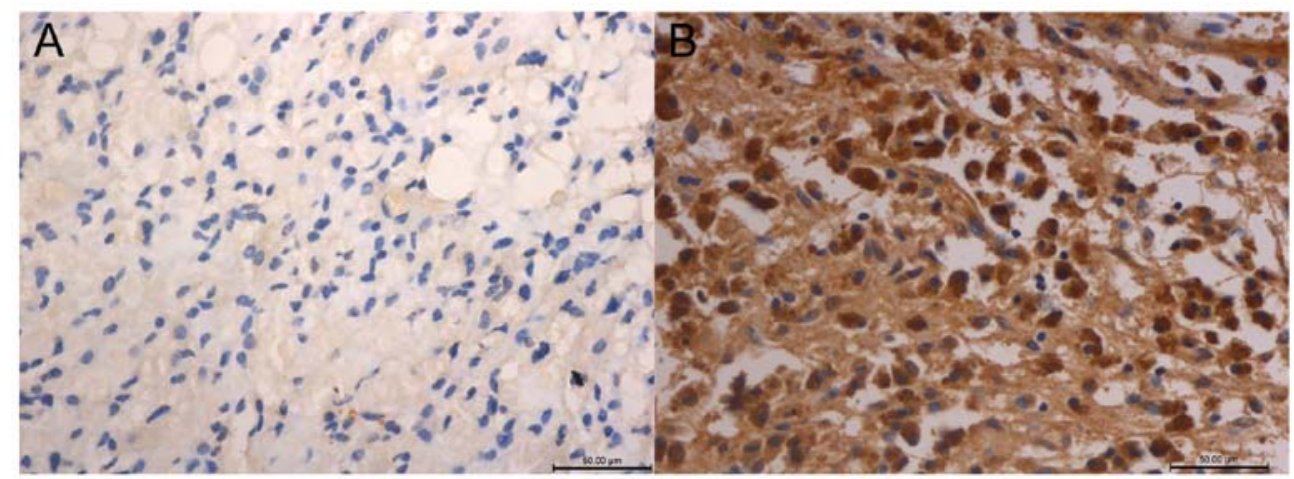

Figure 2. The protein expression of RAB38 was correlated with glioma grade progression. IHC showed RAB38 protein expression ranged from low to high along with grade progression of gliomas. (A) LGG patient (astrocytoma) and (B) HGG patient (glioblastoma). Original magnification, x200.
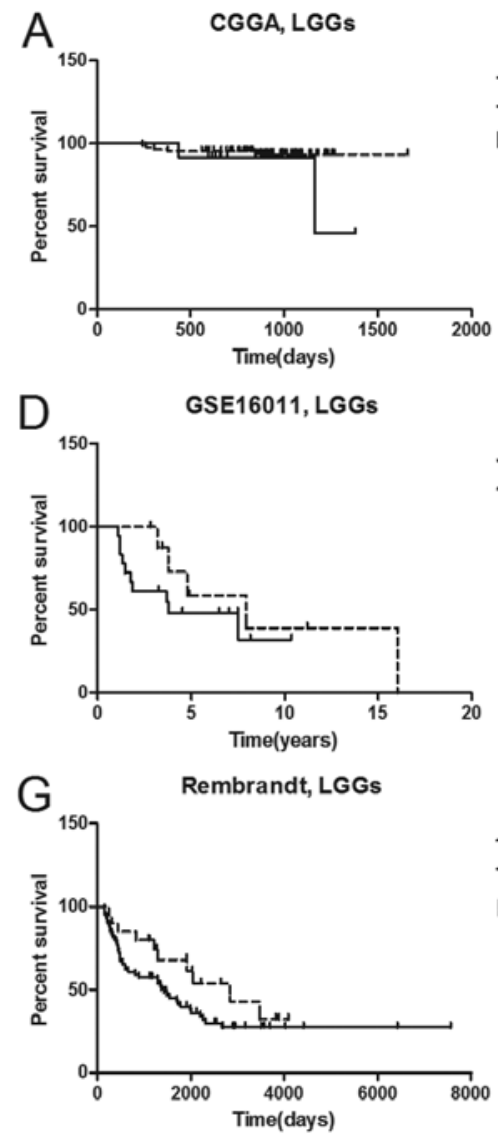
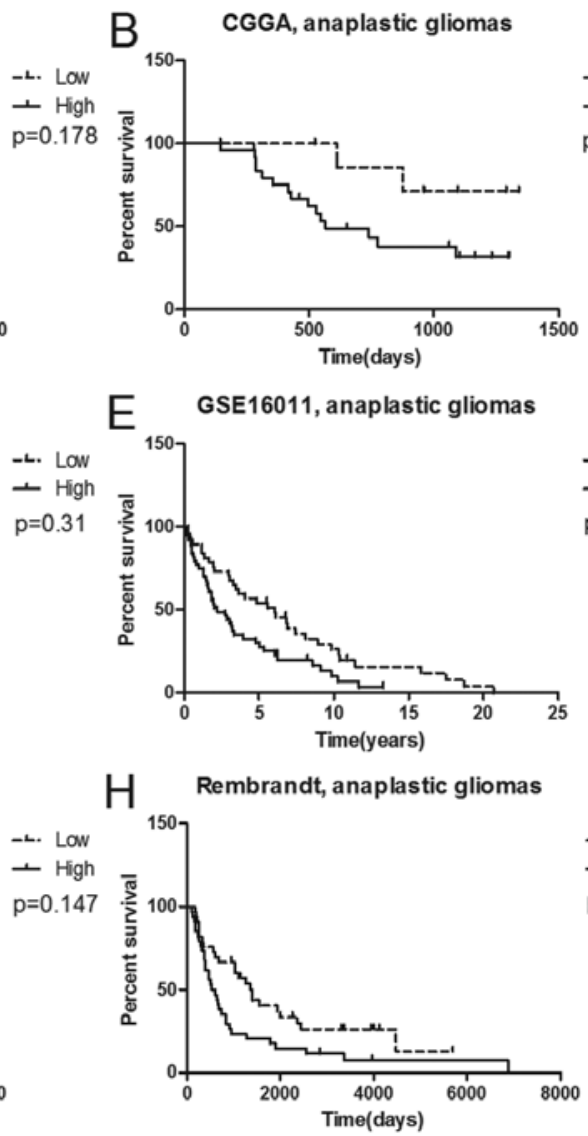
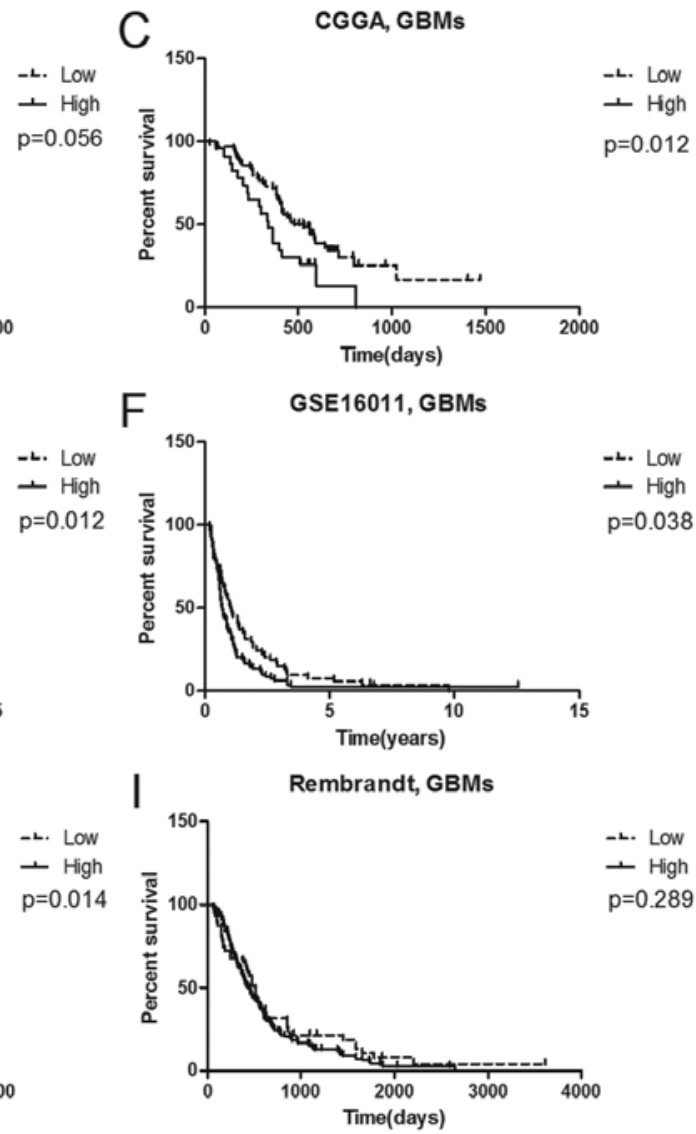

Figure 3. The prognostic analysis of RAB38 in CGGA and validation datasets. P-values were not significant, but LGGs were still divided into two groups (A, D and G). In HGGs, the expression of RAB38 showed significant effect to percent of survival (B, C, E, F and H), except for GBM patients in GSE16011 dataset (I). 
Table II. Gene sets increased in glioblastoma samples with RAB38 overexpression.

\begin{tabular}{|c|c|c|c|c|}
\hline Name & Count & Fold-enrichment & P-value & FDR \\
\hline GO:0005576 extracellular region & 762 & 1.391647 & $2.22 \mathrm{E}-30$ & $3.35 \mathrm{E}-27$ \\
\hline GO:0031226 intrinsic to plasma membrane & 487 & 1.471373 & $1.07 \mathrm{E}-24$ & $1.61 \mathrm{E}-21$ \\
\hline GO:0005887 integral to plasma membrane & 475 & 1.467734 & $8.33 \mathrm{E}-24$ & $1.26 \mathrm{E}-20$ \\
\hline GO:0005886 plasma membrane & 1248 & 1.212935 & $1.04 \mathrm{E}-21$ & $1.57 \mathrm{E}-18$ \\
\hline hsa04080:Neuroactive ligand-receptor interaction & 140 & 2.012199 & $2.96 \mathrm{E}-21$ & $3.68 \mathrm{E}-18$ \\
\hline GO:0031224 intrinsic to membrane & 1711 & 1.1451 & $8.49 \mathrm{E}-19$ & $1.28 \mathrm{E}-15$ \\
\hline GO:0007166 cell surface receptor linked signal transduction & 651 & 1.317325 & $1.72 \mathrm{E}-18$ & $3.29 \mathrm{E}-15$ \\
\hline GO:0016021 integral to membrane & 1648 & 1.142082 & $1.46 \mathrm{E}-16$ & $1.67 \mathrm{E}-13$ \\
\hline GO:0044459 plasma membrane part & 753 & 1.254731 & $2.47 \mathrm{E}-15$ & $3.67 \mathrm{E}-12$ \\
\hline GO:0044421 extracellular region part & 370 & 1.414818 & $2.59 \mathrm{E}-15$ & $3.85 \mathrm{E}-12$ \\
\hline GO:0005576 extracellular region & 762 & 1.391647 & $2.22 \mathrm{E}-30$ & $3.35 \mathrm{E}-27$ \\
\hline GO:0031226 intrinsic to plasma membrane & 487 & 1.471373 & $1.07 \mathrm{E}-24$ & $1.61 \mathrm{E}-21$ \\
\hline
\end{tabular}

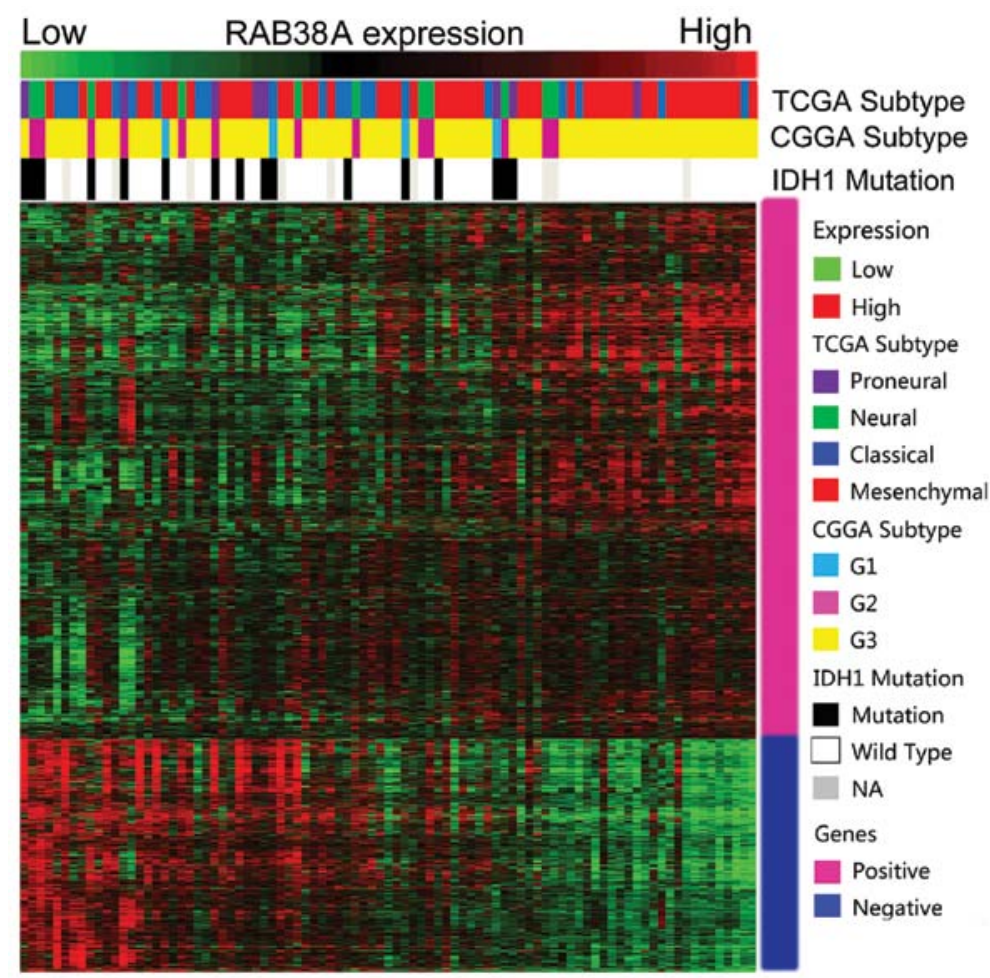

Figure 4. RAB38 confers a mesenchymal, G3 subtypes and IDH1 wild-type preference. For each patient, TCGA and CGGA subtype were annotated as previously reported and listed in the upper part as well as the IDH1 mutation status, which was obtained from CGGA database. The positively and negatively correlated genes are shown in the lower part (marked pink and blue on the right, respectively). NA, not available.

of GSE16011 and Rembrandt cohort (Fig. 3E and H). In GBMs, there was a significantly shorter survival time in high RAB38 expression than those of low in CGGA (Fig. 3C) and GSE16011 (Fig. 3F) except for Rembrandt (Fig. 3I). Therefore, RAB38 was a prognostic marker in anaplastic gliomas and glioblastomas.

RAB38 expression showed a subtype preference. As high RAB38 expression was mainly enriched in HGGs, we screened its expression in different molecular subtypes of GBMs. As previously reported, we annotated the 4 datasets by TCGA and CGGA classification systems by PAM $(2,11)$.
RAB38 with mesenchymal subtype and G3 subtype have high expression level. Patients with IDH1 gene wild-type also showed higher expression of RAB38 than those with mutation IDH1 gene (Fig. 4). The expression difference is shown in Fig. 5.

RAB38 was significantly associated with cell migration. Through Pearson correlation analysis, the significant, positively correlated genes of the data from CGGA (pink marked genes in Fig. 4) were used for GO analysis. The top $10 \mathrm{GO}$ terms listed in Table II show that RAB38 was associated with cell migration. Using a $\mathrm{P}$-value of $<0.001,>40$ biologic processes 

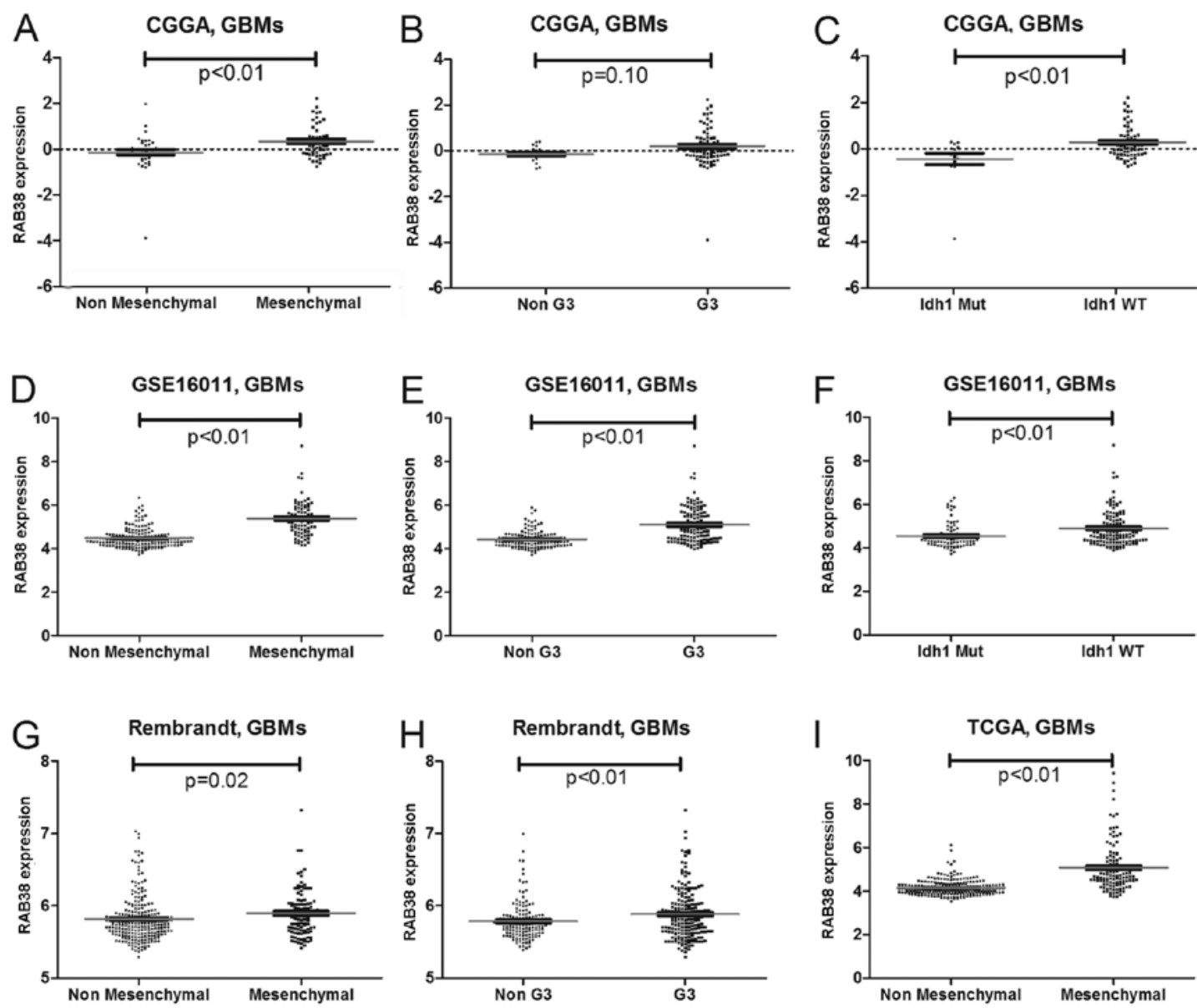

Figure 5. Expression level of RAB38 is significantly different for the different TCGA and CGGA subtypes and IDH1 mutation status. (A, D, G and I) Show that RAB38 has a mesenchymal preference. (B, E and H) Show that RAB38 has a G3 preference. (C and F) Show that patients with IDH1 gene wild-type also have higher expression of RAB38 than those with mutation IDH1 gene.

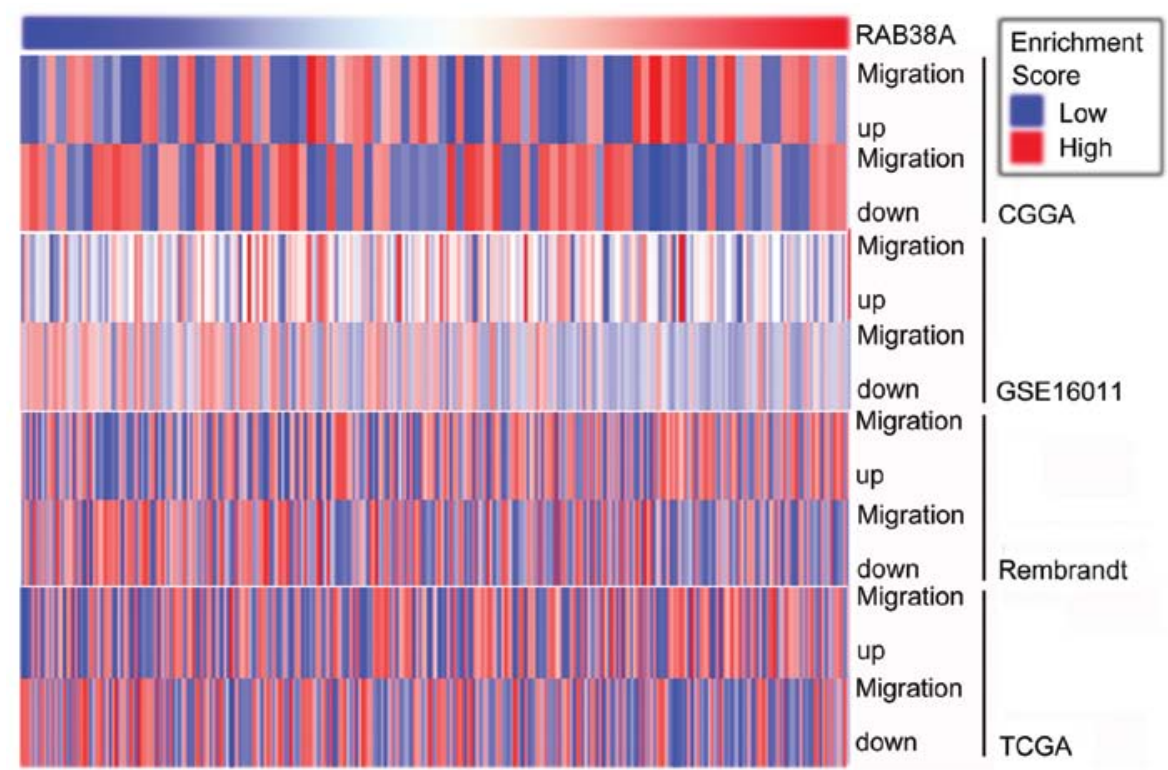

Figure 6. GSVA with RAB38 expression. Gene expression signatures of migration were generated from GOlist. The RAB38 expression increases from left to right. A high enrichment score indicates positive correlation with RAB38 expression and a low enrichment score indicates the reverse.

mainly correlated to cell migration were significantly enriched in the GBMs samples with RAB38 high expression.
Correlation between the migration genes and RAB38. Because RAB38 had a tight association with migration in GO analysis, 
we performed GSVA with RAB38 expression (Fig. 6). Genes up- and downregulated in migration were positively and negatively associated with RAB38 expression in CGGA and the other 3 validated datasets.

\section{Discussion}

In the present study, we found that mRNA level of RAB38 shows high expression in HGGs of CGGA, this was further validated in two published datasets. Protein level of RAB38 was in accordance with mRNA detected by IHC. Prognostic analysis confirmed that high expression of RAB38 indicates short survival time in HGGs. GO and GSVA showed RAB38 was correlated with cell migration. This is the first description of RAB38 in glioma.

Glioma is a diffusely infiltrating brain tumor, with characteristic invasive patterns. The invasiveness is a major cause of therapeutic failure, especially in GBM (15-17). Applying the high throughput technologies, we can explore the significant genes with aberrant expression in tumors and establish their functions.

RAB GTPases mediate vesicle formation and vesicle movement along with actin and tubulin and membrane fusion (18). This process is involved in cell migration with lamellipodial formation. For specific recognition, a vesicle and its target are coded by a unique compliment of RAB GTPases (19). Thus, it is important to understand the intracellular signaling of $\mathrm{RAB}$ proteins in various cells to find new molecular approaches to cancer biology. Some RAB proteins have been reported to be necessary for the adhesion and migration of cancer cells. RAB5 and RAB21, are associated with subunits of $\alpha$-integrin, altering their endosomal traffic and subcellular localizations (20). RAB27a and $\mathrm{RAB} 27 \mathrm{~b}$ were involved in exocytosis of endocrine cells and were associated with the invasive and metastatic potential of breast cancer. There are only few reports on RAB GTPases in glioma $(9,10)$.

RAB38 is a member of the RAB superfamily, its mutation is associated with platelet dense granule storage pool disease $(1,21)$. The effect of RAB38 in tumors has not been reported before. In the present study, we found that RAB38 was highly expressed in HGGs compared to LGGs in mRNA microarray of CGGA (Fig. 1). The different expression levels were validated by microarray data from two other published datasets. The protein level of RAB38 detected by IHC resulted from an independent group of patients. From the above results, we can conclude that RAB38 was a potential marker for grading of gliomas. In the CGGA dataset, significant RAB38 positively correlated genes were analyzed by GO, the result showed RAB38 functions mainly in promoting migration (Table II). GSVA, of the 4 datasets, also showed supporting results.

The contribution of grade progression and survival rate of tumors are very important for the clinical assessment. Improved knowledge of the downstream mediators of RAB38 effects in cancer cells may allow dissection of the different RAB38-induced phenotypes and thereby generation of specific targeted therapies. Analysis of the prognosis in CGGA and 3 other datasets found that patients with higher expression of RAB38 showed a significantly worse prognosis in gliomas.
Hence, RAB38 was shown to be have important effects and to have potential as a prognostic marker and a therapeutic target. We also found the preference of expression of RAB38 in mesenchymal subtype, G3 subtype or IDH1 mutation patients, which was in concordance with the worst prognosis of high RAB38 expression patients.

Collectively, RAB38 expression was increased in HGGs, mesenchymal and G3 subtype and wild-type IDH1. High RAB38 expression confered the worst prognosis in anaplastic gliomas and glioblastomas. RAB38 is a novel, potential biomarker and therapeutic target.

\section{Acknowledgements}

We would like to thank Dr Susan Furness for her critical reading and Professor Y. Chen (Beijing Sanbo Brain Hospital) for IHC technical support. This research was supported by grants from the National High Technology Research and Development Program (no.2012AA02A508), the International Science and Technology Cooperation Program (no. 2012DFA30470), the National 973 Program (no.2011CB707804) and the National Natural Science Foundation of China (no. 81201993).

\section{References}

1. Osanai K and Voelker DR: Analysis and expression of Rab38 in oculocutaneous lung disease. Methods Enzymol 438: 203-215, 2008.

2. Yan W, Zhang W, You G, et al: Molecular classification of gliomas based on whole genome gene expression: a systematic report of 225 samples from the Chinese Glioma Cooperative Group. Neuro Oncol 14: 1432-1440, 2012.

3. Bao Z, Zhang C, Yan W, et al: BMP4, a strong better prognosis predictor, has a subtype preference and cell development association in gliomas. J Transl Med 11: 100, 2013.

4. Recchi C and Seabra MC: Novel functions for Rab GTPases in multiple aspects of tumour progression. Biochem Soc Trans 40: 1398-1403, 2012.

5. He H, Dai F, Yu L, et al: Identification and characterization of nine novel human small GTPases showing variable expressions in liver cancer tissues. Gene Expr 10: 231-242, 2002.

6. Shimada K, Uzawa K, Kato M, et al: Aberrant expression of RAB1A in human tongue cancer. Br J Cancer 92: 1915-1921, 2005.

7. Culine S, Honore N, Closson V, et al: A small GTP-binding protein is frequently overexpressed in peripheral blood mononuclear cells from patients with solid tumours. Eur J Cancer 30A: 670-674, 1994.

8. Yao R, Wang Y, Lubet RA and You M: Differentially expressed genes associated with mouse lung tumor progression. Oncogene 21: 5814-5821, 2002

9. Liu Y, Zhou Y and Zhu K: Inhibition of glioma cell lysosome exocytosis inhibits glioma invasion. PLoS One 7: e45910, 2012 .

10. Overmeyer JH, Young AM, Bhanot H and Maltese WA: A chalcone-related small molecule that induces methuosis, a novel form of non-apoptotic cell death, in glioblastoma cells. Mol Cancer 10: 69, 2011.

11. Verhaak RG, Hoadley KA, Purdom E, et al: Integrated genomic analysis identifies clinically relevant subtypes of glioblastoma characterized by abnormalities in PDGFRA, IDH1, EGFR, and NF1. Cancer Cell 17: 98-110, 2010.

12. Gravendeel LA, Kouwenhoven MC, Gevaert O, et al: Intrinsic gene expression profiles of gliomas are a better predictor of survival than histology. Cancer Res 69: 9065-9072, 2009.

13. Hanzelmann S, Castelo R and Guinney J: GSVA: gene set variation analysis for microarray and RNA-Seq data. BMC Bioinformatics 14: 7, 2013.

14. R: Core Team: A language and enviroment for statistical computing. http://www.R-project.org. R Foundation for Statistical Conputing 2012, Vienna, Austria. 
15. Senft C, Priester M, Polacin M, et al: Inhibition of the JAK-2/ STAT3 signaling pathway impedes the migratory and invasive potential of human glioblastoma cells. J Neurooncol 101: 393-403, 2011.

16. Stupp R, Mason WP, van den Bent MJ, et al: Radiotherapy plus concomitant and adjuvant temozolomide for glioblastoma. $\mathrm{N}$ Engl J Med 352: 987-996, 2005.

17. Wang Y, Chen L, Bao Z, et al: Inhibition of STAT3 reverses alkylator resistance through modulation of the AKT and $\beta$-catenin signaling pathways. Oncol Rep 26: 1173-1180, 2011.
18. Stenmark H and Olkkonen VM: The Rab GTPase family. Genome Biol 2: Reviews 3007, 2001.

19. Barr FA: Rab GTPase function in Golgi trafficking. Semin Cell Dev Biol 20: 780-783, 2009.

20. Subramani D and Alahari SK: Integrin-mediated function of Rab GTPases in cancer progression. Mol Cancer 9: 312, 2010.

21. Ninkovic I, White JG, Rangel-Filho A and Datta YH: The role of Rab38 in platelet dense granule defects. J Thromb Haemost 6: 2143-2151, 2008 